MATEC Web of Conferences 11,02010 (2014)

DOI: $10.1051 /$ matecconf $/ 20141102010$

(C) Owned by the authors, published by EDP Sciences, 2014

\title{
Novel method to measure the soil clay's permeability by laser interferometry
}

\section{Nouvelle méthode pour mesurer la perméabilité des sols argileux par interférométrie laser}

\author{
H. Asselman ${ }^{1}$, A. Daouidi ${ }^{1}$, K. Draoui ${ }^{2}$ et A. Asselman ${ }^{1}$, \\ ${ }^{1}$ Equipe de recherche d'Optique et Photonique, Faculté des Sciences, Tétouan, Maroc. \\ ${ }^{2}$ Laboratoire : Matériaux et systèmes interfaciaux, Faculté des Sciences, Tétouan, Maroc
}

\begin{abstract}
In this study, we'll present a new method to measure the permeability of clay soils (or infiltration rate) by laser interferometry. The device is a modified Michelson interferometer. Indeed on the movable mirror is glued a clay sample in the form of a parallelepiped, dried, of length $\mathrm{L}$, height $\mathrm{H}$ and shaped to retain the drops of water on its surface to control water's content. When injecting a volume of distilled water (a few drops) to the surface of the sample, it swells and moves the mirror, then we can measure the swelling depending on the depth of a clay soil and its permeability. Therefore we measure time that takes water to reach a depth of $Z$ and we determine the infiltration rate: $V=6 \mu \mathrm{m} / \mathrm{s} \pm 3 \%$ in concordance with literal values included between 2,8 and $13,9 \mu \mathrm{m} / \mathrm{s}$ in clayey silt. For this comparison, we used the study of the characterization of the clay fraction to our sample by XRD.
\end{abstract}

\begin{abstract}
Résumé. Dans cette étude nous présentons une nouvelle méthode pour mesurer la perméabilité (ou vitesse d'infiltration) des sols argileux par interférométrie laser. Le dispositif est un interféromètre de Michelson modifié. En effet sur le miroir mobile est collé un échantillon d'argile ayant la forme d'un parallélépipède, séché, de longueur $\mathrm{L}$, de hauteur $\mathrm{H}$ et façonnée de manière à retenir les gouttes d'eau à sa surface pour contrôler la teneur en eau. Lorsqu'on injecte un volume d'eau distillée (quelques dizaines de gouttes) à la surface de l'échantillon, celui-ci se gonfle et le miroir se déplace, on peut ainsi mesurer le gonflement en fonction de la profondeur d'un sol argileux et la perméabilité. Nous mesurons ainsi le temps que met l'eau pour atteindre une profondeur $Z$ et nous déterminons sa vitesse d'infiltration : $V=6 \mu \mathrm{m} / \mathrm{s} \pm 3 \%$ en bon accord avec les valeurs de la littérature qui sont comprises entre 2,8 et $13,9 \mu \mathrm{m} / \mathrm{s}$ dans les limons argileux. Pour cette comparaison nous avons utilisé l'étude de la caractérisation de la fraction argileuse de notre échantillon par DRX .
\end{abstract}

\section{Introduction}

Le dispositif de mesure basé sur l'interférométrie laser a été réalisé au sein de notre équipe de recherche. Il a l'avantage d'obtenir les résultats comme ceux mesurés par l'oedomètre à savoir le potentiel du gonflement et la pression du gonflement. Récemment, après modification du dit dispositif, il nous avons pu mesurer le gonflement en fonction de la profondeur et la perméabilité d'un sol argileux. Ce qui a fait l'objet de deux brevets [1], [2]. Pour obtenir cette vitesse nous utilisons la déformation du matériau (ou gonflement) comme indicateur qui nous permettra de mesurer le temps que met l'eau pour atteindre une profondeur donnée. Il serait donc intéressant de rappeler le phénomène de gonflement et sa complexité [3], [4], [5]. Le matériau argileux présente la particularité de voir sa consistance se modifier en fonction de sa teneur en eau. Lorsque celle-ci augmente on assistera à un phénomène de gonflement du sol, et lorsqu'elle diminue on observe par contre un phénomène de retrait-tassement se traduisant par dommage souvent important sur les ouvrages de génie civil [6], [7]. [8]. [9]. 
Deux grandeurs sont utilisées pour caractériser le gonflement d'un sol:

- Le potentiel de gonflement qui correspond à la variation relative de volume d'un échantillon libre non soumis à des charges mécaniques [10].

- La pression de gonflement qui peut se définir comme étant la pression induite par l'hydratation d'un échantillon confiné dans des conditions isochores ou bien par la pression qu'il faudrait appliquer à l'échantillon ayant gonflé librement pour le ramener à son état initial non déformé [11].

Rappelons que la perméabilité ou la vitesse d'infiltration est le mouvement de l'eau depuis la surface vers la profondeur du sol. L'infiltration est un processus de grande importance économique, elle est vue par l'ingénieur comme un processus de perte et pour l'agriculteur comme un gain. De l'eau infiltrée en bénéficient presque toutes les plantes terrestres et de nombreux animaux; alimente l'eau souterraine et aussi la majorité des courants durant la sécheresse; réduit les inondations et l'érosion du sol. D'autre part, Le stockage souterrain profond des déchets radioactifs pose des problèmes complexes et variés à de nombreux chercheurs, leur objectif fondamental était de mesurer la perméabilité des sols argileux qui doit être la plus faible possible, pour pouvoir estimer la durée de confinement de la radioactivité des déchets [12].

Darcy en 1856 a établi la loi régissant ce phénomène.

Les méthodes expérimentales de mesure de la perméabilité in situ existantes que nous avons relevées dans la littérature sont: système de mesure à niveau variable, système de mesure à niveau constante, Slug Test, puits de pompage... [13]

Les méthodes empiriques rencontrées sont:

-La loi de poiseuille [14]

-Les formules [14], [15]: d'Allen Hazen (correction du a la température), de Schlichter, de Terzaghi et de Loudon....

\section{Dispositif expérimental}

Nos mesures sont basées sur la détection des variations de volume par gonflement qui se traduisent par des variations d'intensité lumineuse au centre des anneaux d'interférences observés à l'œil on n'a pas besoin de certains accessoires comme : la lame compensatrice...

Le dispositif expérimental que nous avons développé est une adaptation de l'interféromètre de Michelson. Il a été utilisé auparavant pour mesurer la dilatation thermique des solides [16].

La différence de marche entre deux franges, brillantesombre, consécutives varie alors de ce qui correspond à une variation de l'épaisseur de la lame d'air de, inférieur au micromètre (environ $0,2 \mu \mathrm{m}$ pour $\lambda=632.8 \mathrm{~nm}$ ) d'où l'intérêt et la sensibilité de notre méthode expérimentale.

L'application originale de ce dispositif pour étudier le phénomène de gonflement des argiles a été présentée pour la première fois par $\mathrm{H}$. Asselman dans 2 ème Journée d'Argile et Développement Durable [3].

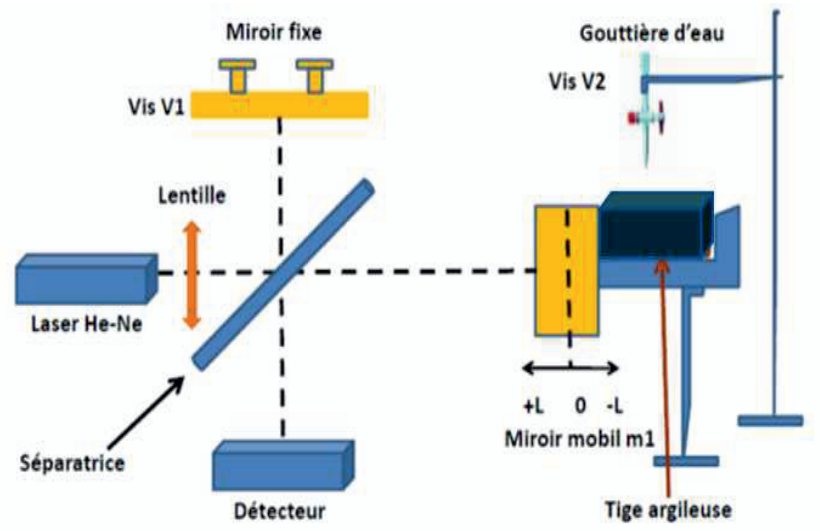

Figure 1: Schéma du dispositif expérimental [1].

Le dispositif expérimental de mesure de la vitesse d'infiltration en profondeur des sols argileux des sols argileux est représenté sur la figure 1. Il comprend:

- Un laser He-Ne de longueur d'onde $632.8 \mathrm{~nm}$ et de puissance $1 \mathrm{~mW}$,

- Une lentille de courte distance focale $(2 \mathrm{~cm})$ pour observer la figure d'interférence

- Un interféromètre de Michelson constitué par une lame séparatrice de transmission 50\%, deux miroirs perpendiculaires: l'un est fixe, orientable dans les plans horizontal et vertical. L'autre est mobile.

- le miroir mobile est relié par l'intermédiaire d'un bras de levier à l'échantillon d'argile ayant la forme d'un parallélépipède, séché, intégré à l'interféromètre et façonné à sa surface longitudinale supérieure, en forme de canal (figure 2) centré, de longueur inférieure à celle $\mathrm{du}$ parallélépipède pour retenir les gouttes d'eau à sa surface et ainsi contrôler la teneur en eau.

- Un écran blanc d'observation des franges d'interférence.

- Le système de contrôle de la teneur en eau est une éprouvette (gouttière) en verre, placée verticalement, graduée en millilitres et remplie d'eau distillée qu'on injecte sur l'argile pour provoquer le gonflement.

- Le système de mesure est constitué d'une caméra (web-cam) vidéo reliée à un ordinateur. Un logiciel associé permettant de suivre et d'enregistrer le défilement des franges d'interférences et leurs comptages $\mathrm{N}$ et le temps.

L'échantillon d'argile de forme parallélépipède (Figure 2 ), de longueur $10 \mathrm{~cm}$, de hauteur $5 \mathrm{~cm}$ et de largeur $1 \mathrm{~cm}$. Le canal à la surface est de longueur $6 \mathrm{~cm}$, de profondeur $4 \mathrm{~mm}$ et de largeur $6 \mathrm{~mm}$. Une série de billes sphériques en acier de diamètres de 3 à $4 \mathrm{~mm}$ légèrement enfoncées et collées de façon équidistante sur les deux faces du parallélépipède. Les deux billes à la même profondeur collées sur les deux extrémités de l'échantillon: l'une reliée au bras de levier de l'interféromètre et l'autre sur le support fixe. 


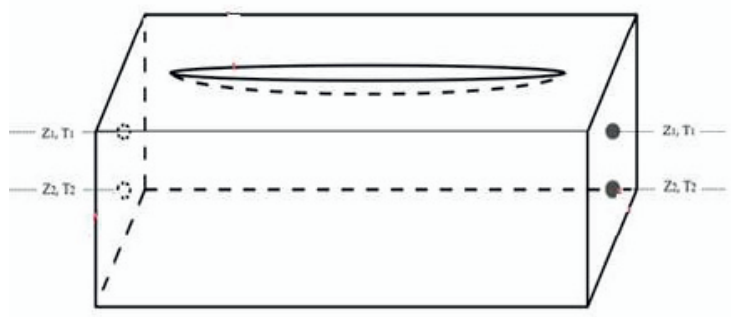

Figure 2: Schéma de l'échantillon argileux

Lorsqu'on injecte un volume d'eau distillée (quelques dizaines de gouttes) à la surface de l'échantillon figure 2 , celui-ci se gonfle et le miroir se déplace. On relève le temps $T_{1}$ quand la déformation par gonflement commence à la profondeur $Z_{1}$ (ce qui indique l'arrivée de l'eau à cette profondeur), se traduisant par le défilement des franges d'interférences. Ensuite on relève le temps $T_{2}$ quand la déformation par gonflement commence à la profondeur $\mathrm{Z}_{2}$. On peut ainsi déterminer la vitesse d'infiltration de l'eau en profondeur d'un sol argileux avec une bonne précision vue la sensibilité de l'interféromètre de Michelson capable de déceler des déplacements inférieurs au micromètre.

Le potentiel de gonflement est donné par :

$$
g(h)=\frac{3 N A}{2 Z}
$$

La perméabilité est donnée par :

$$
v(m / s)=\frac{z 2-Z 1}{T 2-T 1}
$$

\section{Résultats et discussions}

\section{1- Caractérisation de l'échantillon par diffraction des $\mathbf{R X}$}

Dans ce travail, nous avons sélectionné une argile de la région de Tétouan [17], [18]. Plusieurs techniques ont été sollicitées pour la caractérisation physico-chimique de cette argile. La capacité d'échangé cationique CEC a été déterminée par adsorption du cation cobalthexamine. Elle est d'environ $19 \mathrm{meq} / 100 \mathrm{~g}$. L'aire spécifique évaluée par volumétrie d'adsorption d'azote est de $28 \mathrm{~m} 2 / \mathrm{g}$. L'isotherme obtenue est de type IV caractéristique de matériaux mésoporeux d'après la classification de Giles [19]. Cette argile est caractérisée donc par une faible $\mathrm{CEC}$ et une faible aire spécifique. Nous avons ensuite réalisée une étude détaillée par diffraction des rayons $\mathrm{X}$. le résultat obtenu est illustré sur la figure 2 qui représente le diffratogramme des agrégats orientés de la fraction argileuse obtenue après traitement de l'argile par l'acide chlorhydrique afin d'éliminer les carbonates. Nous n'avons pas eu recours à l'élimination de la matière organique puisque sa teneur atteint à peine $0,11 \%$.
Le diagramme de diffraction des rayons $\mathrm{X}$ (Figure 3) révèle la présence des raies $\mathrm{d} 001$ vers $14,5 \AA, 12,1 \AA$ et $7,11 \AA$. Les raies situées vers 3,54 sont attribuée au quartz, minérale secondaire souvent associé aux argiles.

Ces réflexions subissent une légère modification après traitement avec de l'éthylène glycol. Elles apparaissent à des distances de $14,2 \AA, 10 \AA$ et $7,12 \AA$, synonyme à de l'absence de toute trace d'argile gonflante (Figure 4).

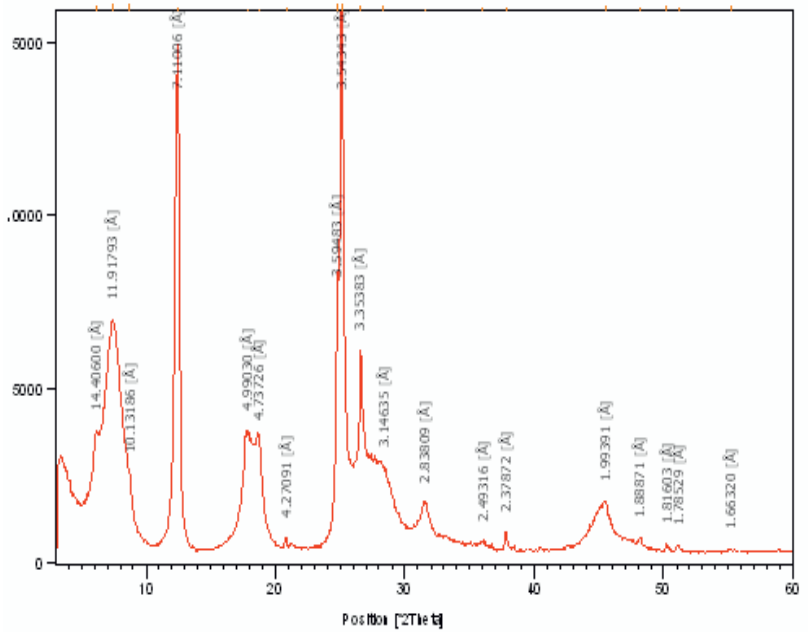

Figure 3: DRX des agrégats orientés de l'argile de Tétouan

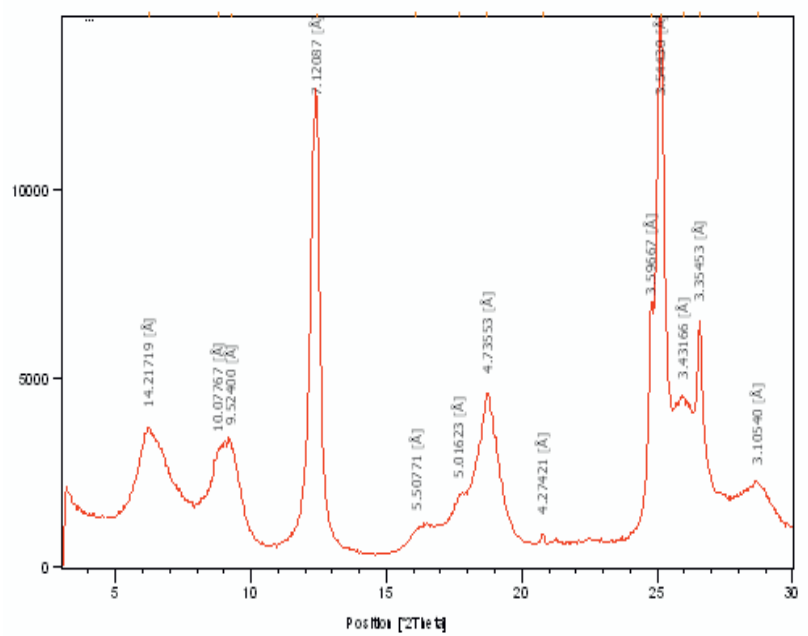

Figure 4:DRX des agrégats orientés de l'argile traités à l'éthylène glycol

Après traitement thermique on note essentiellement la disparition de la raie à 7,11 $\AA$. Ce comportement est attribué aux minéraux de la famille du kaolin dont la structure se détruit à partir de $500^{\circ} \mathrm{C}$. Les raies à $13,9 \AA$ et $10,1 \AA$ (diagramme après traitement thermique) sont relatives à un inter-stratifié Chlorite-Illite. L'argile ANG-T1 est donc un inter-stratifié constituée principalement de trois familles argileuses non gonflantes, la chlorite, l'illite et la kaolinite. Ces résultats sont en bon accord avec la CEC et l'aire spécifique citées ci-dessus. 


\section{2-Mesures de la vitesse d'infiltration}

La méthode de mesure expérimental de la vitesse d'infiltration en profondeur d'un sol argileux a été brevetée par le professeur Hassan Asselman [2].

Cette expérience a été réalisée sur un échantillon de longueur L, de largeur 1 et de hauteur Z (Figure 2). La courbe obtenue (figure 5) est en accord avec les allures des courbes rencontrées dans la littérature [18].

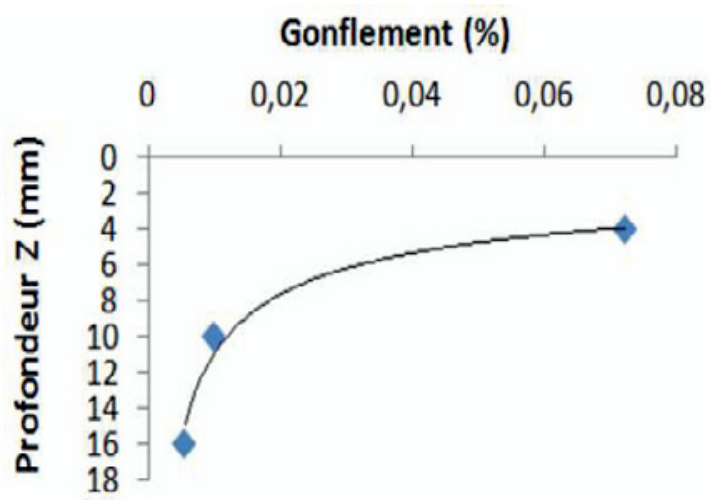

Figure 5 :Gonflement (\%) en Fonction De La Profondeur Z (mm), pour une teneur en eau de 5,3\%

Nous mesurons le temps que met l'eau pour atteindre une profondeur $\mathrm{Z}$ et nous déterminons sa vitesse d'infiltration :

À la $1^{\text {ere }}$ goutte déposé sur le canal à la profondeur $Z_{1}$ le défilement des franges est instantané à cause de la sensibilité de l'interféromètre capable de détecter des déplacements inférieurs au dixième de micromètre et on Relève $T_{1}$. L'incertitude majorée sur le temps de la mise en évidence de la déformation par l'observateur est de l'ordre de $\Delta T=2 \mathrm{~s}$. Après séchage de l'échantillon pendant $24 \mathrm{H}$ dans l'étuve à $105^{\circ} \mathrm{C}$ on déplace les 2 points de contact de l'échantillon à la profondeur $\mathrm{Z}_{2}$. Après un temps $T_{2}$, le défilement reprend et on relève cet instant.

L'écart entre les deux billes successif est mesuré au moyen d'un pied à coulisse et l'incertitude sue la profondeur majorée $\Delta \mathrm{Z}=0.1 \mathrm{~mm}$ ce qui nous conduit à une précision globale sue la perméabilité ne dépassant pas les $3 \%$. Pour améliorer cette précision il suffira d'augmenter la profondeur.

Pour cette expérience nous avons fixé un nombre de 30 gouttes injectés à la surface de l'échantillon.

Nous avons ainsi obtenu $\mathrm{V}=6 \mu \mathrm{m} / \mathrm{s} \pm 3 \%$. On a vérifier qu'elle est constante lorsqu'on change l'intervalle de profondeur entre $Z_{2}$ et $Z_{3}$. d'après la figure : $Z 1=4 \mathrm{~mm}$, $Z 2=10 \mathrm{~mm}$ et $Z 3=16 \mathrm{~mm}$. Notre résultat est en bon accord avec les valeurs de la littérature (mesurées par la méthode courante à niveau variable la plus utilisée) [20] qui sont comprises entre 2,8 et $13,9 \mu \mathrm{m} / \mathrm{s}$ dans les limons argileux (Tableau 1).

\begin{tabular}{|l|c|}
\hline \multicolumn{1}{|c|}{ Type de sol } & $\begin{array}{c}\text { Vitesse d'infiltration } \\
(\mu \mathrm{m} / \mathrm{s})\end{array}$ \\
\hline Sableux & $>27,8$ \\
\hline Leger (limon sableux) & $13,9-27,8$ \\
\hline Moyen (Limon argileux) & $2,8-13,9$ \\
\hline Lourd (Argileux) & $1,4-2,8$ \\
\hline Très lourds & $0,28-1,4$ \\
\hline
\end{tabular}

Tableau 1 : les valeurs de la vitesse d'infiltration selon le type de sol, par la méthode à niveau variable [20].

En effet la granulométrie de notre échantillon (Tableau 2) montre qu'il s'agit d'un limon argileux. Dans la fraction argileuse de $32.1 \%$ nous avons plus de Chlorite que d'Illite [17]. Ces mesures ont été réalisées au laboratoire de Salamanca IRNASA (CSIC) - Espagne Les valeurs de la vitesse d'infiltration [20] présentées dans le tableau 1 sont des estimations seulement et la précision dépasse les $50 \%$.

\begin{tabular}{|l|l|}
\hline $\begin{array}{c}\text { Granulométrie de } \\
\text { notre échantillon }\end{array}$ & \multicolumn{1}{|c|}{ Matériaux } \\
\hline $\mathbf{3 2 . 1 \%}$ & Argiles : Chlorite, Illite \\
\hline $\mathbf{2 7 . 5 \%}$ & $\begin{array}{l}\text { Limon : mélange Silice + } \\
\text { Argile }\end{array}$ \\
\hline $\mathbf{3 6 . 5 \%}$ & Sable \\
\hline
\end{tabular}

Tableau 2 : La granulométrie de notre échantillon [17].

\section{Conclusions}

Dans ce travail nous avons effectué une étude expérimentale originale de l'argile de la région de Tétouan par une nouvelle méthode basée sur l'interférométrie laser qui nous permet de mesurer d'une manière très précise le gonflement en fonction de la profondeur d'un sol argileux et la vitesse d'infiltration de l'eau.

Cette nouvelle technique se distingue des autres méthodes : elle permet de mesurer le gonflement des argiles en un temps record (environ $20 \mathrm{~min}$ ) au lieu de quelques semaines, avec une bonne précision (environ $3 \%$ ) et une bonne sensibilité $(0.2 \mu \mathrm{m})$.

En outre elle constitue une nouvelle méthode expérimentale pour déterminer la perméabilité (ou vitesse d'infiltration) de l'eau dans les sols argileux avec une bonne précision inferieur à $3 \%$ contrairement à celles obtenues par d'autres méthodes expérimentales et empiriques. Notre résultat : $\mathrm{V}=6 \mu \mathrm{m} / \mathrm{s} \pm 3 \%$ est en bon accord avec les valeurs estimées de la littérature, qui sont comprises entre 2,8 et $13,9 \mu \mathrm{m} / \mathrm{s}$ pour les limons argileux. 


\section{Références}

1. Brevet d'Invention $\mathrm{N}^{\circ} 35229$, « Nouvelle méthode optique pour mesurer le gonflement des argiles par interférométrie laser », déposé par Hassan ASSELMAN, le 18 septembre 2012 à l'OPMIC (Office Marocaine de la Propriété Industrielle et Commerciale), Casablanca.

2. Brevet d'Invention $\mathrm{N}^{\circ} 35556$, «Dispositif de mesure de la vitesse d'infiltration de l'eau en profondeur d'un sol argileux", déposé par Hassan ASSELMAN le 10 janvier 2013 à l'OPMIC (Office Marocaine de la Propriété Industrielle et Commerciale), Casablanca.

3. H. Asselman. Deuxième Journée D'argiles et Développement Durable- Tétouan 26 mai 2010.

4. H. Asselman, A. Bakkali , M. Ajdour et M. Essaaidi. Novel experimental method to determine clay soil free swelling . MATEC Web of Conferences 2, (2012)

5. Alonso E.E., Gens A., Hight D.W. General report. Special problem soils. Proceedings of the 9th European Conference on Soil Mechanics and Foundation Engineering, Dublin, Vol.3, 109871146. (1987).

6. D. Magnan Caractérisation in situ des sols gonflants: l'essai Expansol. Thèse de doctorat, Université Joseph Fourier, Grenoble, 190p. (1993).

7. Hossein Nowamooz, Farimah Masrouri. Hydromechanical behaviour of an expansive bentonite/silt mixture in cyclic suction-controlled drying and wetting tests. Engineering Geology, 10 May (2008)

8. H. Asselman , A. Khamlichi, A. Bakkali , et M. Essaaidi. Nouvelle méthode expérimentale pour déterminer le potentiel de gonflement libre des sols argileux. 10eme Congrès de Mécanique. Oujda du 19 au 22 avril (2011)

9. Muzahim Al-Mukhtar, Jean-Claude Touray, Faiza Bergaya. Une argile modèle pour l'étude du gonflement des sols argileux : la laponite-Na de synthèse.Académie des sciences, Elsevier, Paris. Juin (1999).

10. H. Guiras-Skandaji Déformabilité des sols argileux non saturés : étude expérimentale et application à la modélisation. Thèse de Doctorat, Institut National Polytechnique de Lorraine, Ecole Nationale Supérieure de Géologie, Nancy, 315p. (1996).

11. Tabani $\mathrm{P}$ Transfert hydrique dans des sols déformables. Thèse de Doctorat, Institut Nationale Polytechnique de Lorraine, Ecole Nationale Supérieure de Géologie, Nancy, 17. (1999).

12. Jérôme GAOMBALET, Thèse de doctorat de l'école polytechnique, " le gonflement des argiles et ses effets sur les ouvrages souterrains de stockage ». (2004)
13. Christopher, Barry R, De Jong, Jason ; Maayine, Paul W - Manual on Subsurface InvestigationsNational Highway Institute- Washington, Dc (2001).

14. Terzaghi, Karl; Pechk, Ralph Principios Fundamentales de Mecanica de SuelosCompania Editorial Continental S.A, Mexico. (1973, 2 ${ }^{\mathrm{a}}$ Edicion)-

15. Juarez Badillo, Eulalio; Rodriguez, Alfonso R Mecanica De los suelos-TOMO III- Editorial LIMUSA, Mexico. -(1980)-

16. H. Asselman, El Addad M. Congrès COMPLES'2K7. Energie et Environnement. 1920 Octobre 2007. P 178-181 ENSA Agadir, Maroc (2007).

17. H. Azejjel, C. del Hoyo, K. Draoui; M.S. Rodríguez-Cruz, M.J. Sánchez-Martín Natural and modified clays from Morocco as sorbents of ionizable herbicides in aqueous medium. Desalination, volume 249, issue 3, 25 december (2009), pages 1151-1158

18. H. Azejel, Synthèse et caractérisation des argiles marocaines organophiles. Application à la rétention des pesticides, Thèse de Doctorat, Faculté des Sciences de Tétouan; juin (2011).

19. Giles C. H., MacEwan T. H., Nakhwa S. N. et Smith D. Studies in adsorption: Part IX. A system of classification of solution adsorption isothems and its use on diagnosis of adsorption mechanisms and in measurement of specific areas of soils. Journal of Chemical Society, 111, 39733993. (1960)

20. Laoura Elena Maderey Rascóncon la colaboración de Arturo Jiménez Román Principios de hidrogeografía. Estudio del ciclo hidrológico Serie Textos Universitarios, Instituto de GeografíaUniversidad Nacional Autónoma de MéxicoSerie Textos Universitarios, Núm.1, (2005). 